\title{
Pokémon Go and The Law: Privacy, Intellectual Property, and Other Legal
}

\section{Concerns}

JULY 19, 2016 BY TIFFANY LI

Pokémon Go made 22-year-old Kyrie Tompkins fall and twist her ankle. "[The game] vibrated to let me know there was something nearby and I looked up and just fell in a hole," she told local news outlet WHEC 10.

So far, no one has sued Niantic or The Pokémon Company for injuries suffered while playing Pokémon Go. But it's only a matter of time before the first big Pokémon Go related injury, whether that comes in the form of a pedestrian drowning while catching a Magikarp (the most embarrassing possible injury) or a car accident caused by a distracted driver playing the game.

Before the first lawsuits arrive, here's a brief analysis of some of the legal issues involved with the new hit mobile game.

\section{LIABILITY FOR INJURIES}

A few minor injuries have already happened to Pokémon Go players. If a serious accident does occur, injured players can look to legal precedent from Snapchat-related car crashes. 
The Snapchat claimants sued on a theory of product liability, essentially stating that Snapchat created a product that had inherent risks of foreseeable harm to consumers and/or released a product without sufficient warnings against potential harms. Similarly, Pokémon Go players could argue that it's predictable that players would stare at their phones while walking distractedly, ignoring natural hazards and oncoming cars.

However, many of the Snapchat lawsuits center on Snapchat's speed filter encouraging drivers to Snap while driving. No such filter exists for Pokémon Go. In fact, the game is not playable if the player is moving above a certain speed.

Furthermore, Pokémon Go has a number of warnings and safeguards against playing while driving or walking at dangerous speeds. A full-screen warning is displayed during loading that warns users against distracted playing. The game's Terms of Service also includes disclaimers against liability and a warning about Safe Play: "During game play, please be aware of your surroundings and play safely."

\section{$\underline{\text { PRIVACY }}$}

\section{Let's start with the good:}

Niantic has properly covered the basic privacy law requirements. The app includes clearly visible links to their privacy policy, which is also written clearly and (relatively) understandably. 
The privacy policy includes the necessary information, for both U.S. and E.U. users. Niantic has taken the necessary steps to protect children's privacy as well.

\section{And now for the possibly less-good:}

Early on, players noticed a concerning privacy setting that effectively allowed Niantic access and control over players' Google accounts. Niantic quickly fixed this problem and removed the access controls in an update. It's likely that this level of Google account control was a holdover from the days when Niantic was still under the Google umbrella. I would chalk this up as a wash for Niantic, as the privacy concern was resolved fairly quickly.

Now, the real concern here is that the app takes in a lot of information. A lot of information. Some of it is personally identifiable information (like your name and email address). Some of it is user-submitted, like names you give to the forty Rattattas you catch in one day, because even the Pokémon in Manhattan are mostly rats and pigeons. Pokemon Go collects so much information that Senator Al Franken was inspired to publish a letter to Niantic demanding more clarity on the game's privacy protections.

The most concerning privacy issue with this app is the constant tracking of location data. Some of these concerns were already noted, to less fanfare, with the release of Ingress, the precursor to Pokémon Go. By agreeing to the Pokémon Go privacy policy, you explicitly agree to allow Niantic to track your location any time you use the app. Most players leave the app open at all times, waiting for that sweet, sweet buzz of a new wild Pokémon appearing. This means that, 
effectively, you give permission for Niantic to track your movements all day, every day, wherever you go.

Niantic also does not provide much information on how your data can be shared. The privacy policy allows Niantic to "share aggregated information and non-identifying information with third parties for research and analysis, demographic profiling, and other similar purposes." This means data on your daily commute can be sold to marketing companies to better market to you, the consumer. Niantic promises not to share any of this data without aggregating the data (grouping it together with others' data) and stripping it of identifying information (your name, email, etc.).

These problems may sound concerning at first. However, if you are upset about your information being monetized, it's probably too late. Pokémon Go is a low level offender in this category, as basically all the apps on your phone and computer can and do track you now. (As do your store customer loyalty cards, your credit cards, your television, your, your thermostat, and sometimes even your clothing.)

The app has access to a lot of data, including location data, movement data, and potentially photographs. This information, in the wrong hands, could lead to a privacy nightmare for users.

\section{INTELLECTUAL PROPERTY}


Pokémon Go's locations use photos and names taken from Ingress, which includes crowdsourced photos, names, and locations crowdsourced by Ingress users. Both games operate based on data from Google Maps.

Whether Niantic can use photos of landmarks in the Pokémon Go game is a legal grey area. In the United States, there exists a "freedom of panorama" exception to copyright law. This exception allows for free use of photos and videos of buildings (and sometimes sculptures and other works) that are located in public spaces. For example, a mural on a building would fall under this exception, and photographers could freely take photos of that mural. If the mural became a Pokéstop in Pokémon Go, the app could freely use a photo of that mural in the app. However, freedom of panorama sometimes does not extend to other works of art visible in public spaces. This could include sculptures and artworks, some of which are used as locations with photos in Pokémon Go.

Another potential intellectual property issue is whether trademark owners can sue for Niantic's use of their marks in the game. These trademarks could include, say, the name of a store or mural used as a landmark. However, use of a trademark to denote location or origin is generally legally permissible as "fair use" under U.S. trademark law.

\section{AUGMENTED REALITY}

One of the most popular features of Pokémon Go is the app's use of augmented reality. When a wild Pokémon appears, you can choose to see the Pokémon actually in your real life 
surroundings (as seen through your phone camera). You then attempt to catch it (in my case, mostly after many, many tries), while the Pokémon dances on-screen in your kitchen sink, on your friend's head, on the dashboard of your car, etc.

Augmented reality is not a new concept, but use in games is still not widespread. However, there are some intriguing legal issues related to augmented reality, as noted in this primer by the University of Washington's Tech Policy Lab. It remains to be seen how augmented reality apps, including Pokémon Go, affect the legal landscape of what the Tech Policy Lab notes as the major legal issues at play with AR: "privacy, free speech, and intellectual property as well as novel forms of distraction and discrimination."

\section{VIRTUAL CURRENCY}

Currently, players can purchase in-game items through a micro-transaction process that should be familiar to any mobile game consumer. Eventually, the game will likely allow players to trade Pokémon as well, and perhaps items. The more the game allows for purchases and trades, whether between player and in-game store or between player and player, the more potential there is for legal issues regarding virtual currencies in mobile gaming. There is a wide range of legal issues at play with virtual currencies, from consumer fraud to international money laundering to material support of terrorism (seriously).

\section{TRESPASS}


Niantic's Terms of Service explicitly calls out trespass. Trespass, generally, is the act of knowingly entering another person's property without permission. By using the app, you agree to not commit trespass, and Niantic excuses themselves from liability concerning your or any other users' trespass onto private property. Disclaimers, especially in fine print Terms of Service like this, are not bulletproof protections for companies. It's possible that, say, a homeowner could sue Niantic for negligence in allowing for trespass to occur, but it would be much easier (though probably much less lucrative) to sue the trespasser directly.

However, trespass may not be a big issue with this game. Generally, if you see a Pokestop, gym, or wild Pokémon, you can interact with the location without being right next to it. Of course, there may be some exceptions. Many of the locations were taken directly for the crowdsourced landmarks of Ingress, and some of these gyms and Pokestops may be within the bounds of privately owned property. Let's be clear: Even if a Mewtwo appears in your neighbor's yard, you are still not allowed to trespass on private property to get it.

\section{CONCLUSION}

This is not an exhaustive list of every legal possibility inherent in the Pokémon Go world. These are just some of the legal issues at play when users, well, play. While this may seem like a long list of potential legal problems, the reality is that any mobile game will have a host of legal issues involved. What's important here is that users are informed of their rights, which Niantic does its best to achieve through in-game warnings and explicit provisions in their Terms of Service and Privacy Policy. 
While I can't give you legal advice here, I can say that, as a technology attorney with substantial experience researching privacy and intellectual property, I personally have no qualms playing the game. Just try not to fall off a cliff while playing. It's not worth it, unless there really is a Mewtwo at the bottom of that cliff - in which case, where is that cliff, exactly?

\section{Recommended Reading:}

1. Beth Hill, IAPP Privacy Advisor, "The important privacy lessons from 'Pokemon Go'"

2. Jedidiah Bracy, IAPP Privacy Advisor, "Pokémon GO, augmented reality, and privacy"

3. Gabriella Ziccarelli, Baltimore Sun, "The Price of Pokemon Go"

4. James Rogers, Fox News, “Death by Pokemon? Public safety fears mount as 'Pokemon GO' craze continues"

5. Rachel Dicker, US News \& World Report, "Could Pokemon Go Break Election Laws?'

6. National Constitution Center, "Pokemon Go shines new attention on trespass laws"

7. Keith Lee, Associate’s Mind, “Is Pokemon Go Illegal?"

8. Brian D. Wassom, Hollywood Reporter, “How Pokémon GO Players Could Run Into RealLife Legal Problems"

9. Natasha Lomas, TechCrunch, “Pokemon Go T\&Cs strip users of legal rights"

10. University of Washington Tech Policy Lab, Augmented Reality Technology Policy Primer 